\title{
The Effects of Repeated Sprints on the Kinematics of 3-Point Shooting in Basketball
}

\author{
by \\ Jean Slawinski ${ }^{1}$ Julien Louis' ${ }^{2}$ Julien Poli ${ }^{1}$,Eve Tiollier ${ }^{3}$, Charles Khazoom ${ }^{4}$, \\ Daniel Dinu ${ }^{3}$
}

\begin{abstract}
Fatigue modifies the kinematics of various sports-related movements. Basketball induces fatigue, however, the effects of fatigue on the kinematics of shooting have never been studied. This study analysed the effects of fatigue induced by repeated sprints on the kinematics of 3-point shooting (3PS) in young, elite basketball players (U18 level). 3D joint angles were calculated at the maximum and minimum heights of the centre of mass during 3PS, using inertial measurement units (Biomech system, Xsens Technologies BV, Enschede, The Netherlands). Height, velocity and the angle of the ball at the time of release were extrapolated from the wrist joint angles. All players performed four 3PS actions in dynamic conditions before and after a fatigue protocol at $70 \%$ of their maximal exercise capacity. The fatigue protocol consisted of a shuttle test with repeated 20-m sprints interspersed with sets of 5 jumps. There was no change in the kinematics of 3PS $(p>0.05)$, or the ball release variables $(p>0.05)$ following the fatigue protocol. This suggests that elite basketball players are able to cope with physical fatigue while performing coordinated movements such as 3PS.
\end{abstract}

Key words: biomechanics, team sport, kinematics, elite players, fatigue.

\section{Introduction}

Basketball is a team sport that involves speed, acceleration, changes of direction and precision (Ben Abdelkrim et al., 2007; Montgomery et al., 2010; Mikołajec et al. 2012). Actions are executed and repeated at a high intensity, causing gradual fatigue. An elite basketball game can elicit up to $90 \%$ of the maximal heart rate, and blood lactate concentration can reach $\sim 6 \mathrm{mmol} \cdot \mathrm{L}^{-1}$ at half time and after the game (Ben Abdelkrim et al., 2010; Matthew and Delextrat, 2009). Moreover, recent data from the 2012 Olympic Games and from other international events, world or European championships, showed that a game is generally won with a lead of at least 5 points. A tight score at the end of the game increases stress on players to make precise shots and significantly increases both mental and physical constraints (Bar-Eli et al., 2006). In addition, new game rules initiated in 2000 such as the reduction of offensive time from 30 to $24 \mathrm{~s}$ require more precise and rapid actions, significantly increasing the physical demand. The physical ability to perform repeated sprints at high intensity until the end of the game plays a central role in competition and can determine the final outcome (Girard et al., 2011; Lyons et al., 2006; Mikołajec et al., 2012). Analysis of the 74 games of the 2010 basketball World Championship showed that the average rate of 3point shots (3PS) during the last $5 \mathrm{~min}$ of a game was significantly higher in winning teams than losing teams (Malarranha et al., 2013). In general,

\footnotetext{
1 - UFRSTAPS - Centre de recherche sur le sport et le mouvement - EA 2931. Université de Paris Nanterre, France.

2 - Liverpool John Moores University - Research Institute for Sport and Exercise Sciences - Liverpool - United Kingdom.

3 - French National Institute of Sport (INSEP), Research Department, Laboratory Sport, Expertise and Performance (EA 7370),

Paris, France.

4 - Université de Sherbrooke - 2500 boul. de l'Université, Sherbrooke, Canada, J1K 2R1.
} 
3PS represents $16 \%$ of points scored in a game and determine the final outcome to a great degree (Guo et al., 2004). However, recent studies have shown that repeated sprint ability was significantly reduced after a match, and 3PS accuracy decreased by more than $20 \%$ at $80 \%$ of the maximal heart rate (HRmax) (Delextrat et al., 2013; Padulo et al., 2015). Muscle factors (i.e. muscle excitability, energy supply and metabolite accumulation), neural factors (i.e. neural drive, muscle recruitment strategies) and stiffness regulation could explain the decrease of performance during repeated sprints (Girard et al., 2011). A recent review of the literature on basketball jump shots (Okazaki et al., 2015) suggested that these different factors may influence shooting effectiveness. However, the actual effect of physical fatigue on performance of basketball jump shots remains unclear because results of the available studies are contradictory. Several studies have shown that fatigue of the primary muscle involved in a motor task such as the vertical jump, leads to an increased activation of synergistic muscles not normally involved in the task (Rodacki et al., 2002). Thus, if the main muscles involved in 3PS are fatigued, the kinematics of the shot should be modified. Fatigue could affect both the lower limb muscles that contribute to the jump and the upper limb muscles that contribute to ball release. If body kinematics are altered (i.e. ankle, knee, hip, shoulder, elbow, wrist angles and movement of the centre of mass), ball kinematics are likely to be sub-optimal since a successful shot requires a specific combination of a ball release angle, height and velocity (Hamilton and Reinschmidt, 1997; Okazaki et al., 2015).

Fatigue is an important element in basketball games, yet only a few studies have analysed its effect on performance and biomechanics of a successful shot (Erculj and Supej, 2009; Uygur et al., 2010). They used video analysis and reported contradictory results for 3PS. Erculj and Supej (2009) found that fatigue significantly reduced the maximal height of the centre of mass and altered shoulder and elbow joint angles, whereas Uygur et al. (2010) did not find any effect of fatigue on upper and lower limb joint kinematics. Both studies used exhaustion protocols (i.e. combinations of sprints, jumps and direction changes) which elicited $100 \%$ of the players' maximal capacity (i.e. $100 \%$ of the maximal heart rate or exhaustion). Both protocols were more exhausting than a real basketball match, during which players generally reach from 75 to $85 \%$ of their HRmax (Delextrat et al., 2013; Matthew and Delextrat, 2009; McInnes et al., 1995; Padulo et al., 2015). Studies that mimic the physical constraints of a basketball game need to be carried out to understand the impact of fatigue on shooting performance.

The aim of the present study was therefore to assess the impact of physical fatigue on upper and lower limb joint kinematics (i.e. 3D angles of the ankle, knee, hip, shoulder, elbow and wrist, and the height of the centre of mass) and on variables related to ball release (i.e. height, velocity and angle of the ball at release time) during 3PS. Particular attention was given to implementing a fatiguing exercise protocol (eliciting around $80 \%$ of the maximal heart rate) that mimicked the level of fatigue typically encountered in a match. We hypothesized that fatigue would result in an alteration of kinematic variables, which would reduce the effectiveness and accuracy performance of 3PS.

\section{Methods}

\section{Participants}

Ten young, right-handed basketball players (age: $16.3 \pm 1.2$ years; body height: $1.90 \pm$ $0.13 \mathrm{~m}$; body mass: $76 \pm 12.2 \mathrm{~kg}$ ) volunteered to participate in the study. These young elite athletes in the U18 squad (6 males and 4 females) were affiliated to the basketball academy of the National Institute of Sport, Expertise and Performance (INSEP, Paris, France). All participants were free of injuries at the time of data collection. This study was approved by the French National Basketball Association and carried out in accordance with the Declaration of Helsinki. All the participants were informed of the objectives and risks of the study and the parent or legal guardian signed an informed consent form before the commencement of the study.

Measures

Two testing sessions (over two days) were organized in an indoor basketball gym of the French Institute of Sport. The purpose of the first session was to determine each participant's time to exhaustion $\left(\mathrm{T}_{\mathrm{ExEx}}\right)$, so that the kinematics of shooting could be recorded at $70 \%$ of TExEx during the second session.

During the first session, the participants 
carried out a volitional exhausting exercise protocol $\left(E_{x} E_{x}\right)$. This fatigue protocol consisted of repeated $20 \mathrm{~m}$ sprints with 5 consecutive vertical jumps at both ends of the sprints (Chappell, 2005; Uygur et al., 2010). Participants were instructed to accelerate/decelerate maximally during the sprints and to jump as high as possible at each end, until exhaustion. Time to exhaustion and the heart rate (HREx) were measured (Suunto T6, Vantaa, Finland) during the test. Each $20 \mathrm{~m}$ sprint was timed (Microgate, Bolzano, Italy). The Borg scale (6-20) was used to evaluate the level of fatigue perceived during the exercise protocol (Borg, 1982).

During the second session (at least $48 \mathrm{~h}$ after the first session), the participants were asked to perform series of four 3PS actions under dynamic conditions. The players ran a few steps, received the ball and shot at a distance of $6.75 \mathrm{~m}$. Any shot that touched the rim or the backboard was considered unsuccessful (Miller and Bartlett, 1996; Rojas et al., 2000). In order to reproduce game conditions, the second step consisted of the $E_{x} E_{x}$, but only until $70 \%$ of $\mathrm{T}_{\mathrm{ExEx}}\left(\mathrm{T}_{\mathrm{ExE} \times 70 \%}\right)$. At the end of the $E_{x} E_{x}$, four other 3PS actions were performed by each participant. During the second session, the players wore a MVN Biomech suit (Xsens Technologies BV, Enschede, The Netherlands). This suit is composed of 17 miniature inertial measurement units (nanotechnology inertial measurement units, nIMU) strapped to the body. Each nIMU contains three gyroscopes, three accelerometers and three magnetometers in a $35 \mathrm{~g}$ box about the size of a matchbox. Each nIMU captures the 6-degrees-of-freedom of the body segment to which it is fixed, in real time at a sampling frequency of $120 \mathrm{~Hz}$.

\section{Design and Procedures}

Kinematic variables

Firstly, the lowest and highest positions of the centre of mass (CoM) were identified and its vertical displacement was calculated. Then, joint angles (abduction/adduction, internal/external and flexion/extension, depending on the joint) were calculated for the ankle, knee, hip, shoulder, elbow and wrist on the throwing side, from the time when the CoM was at its lowest position to the time when the ball was released. Figure 1 depicts the flexion/extension angles of all these joints. Kinematic analysis was performed with software provided by MVN Biomech and a customized MatLab ${ }^{\mathrm{TM}}$ program was used to calculate the elbow, trunk, knee, and ankle joint angles using a newton euler method.

Ball release variables

Release ( $\left.t_{\text {release}}\right)$ was considered to occur when the orientation of the hand was closest to vertical. The release variables of the ball were estimated by first modeling the hand and the fingers as a single rigid segment, termed BC, and by assuming that the ball rolled on the fingers without slipping, with point C (Meriam and Kraige, 2012) being the contact point of the hand with the ball at trelease (Figure 2). Wrist velocity ( $\vec{v}_{\text {wrist }}$ ) and hand angular velocity $\left(\vec{\omega}_{\text {hand }}\right)$, along with length and orientation of the fingers $\left(\vec{p}_{\text {fingers }}\right)$ were calculated by the MVN Biomech system. The velocity of the fingers $\vec{v}_{\text {fingers }}$ at trelease was thus computed using equation 1 .

$$
\vec{v}_{\text {fingers }}=\vec{v}_{\text {wrist }}+\vec{\omega}_{\text {hand }} \cdot \vec{p}_{\text {fingers }}
$$

With the ball's angular velocity $\vec{\omega}_{\text {ball }}$, the release velocity of the ball could be calculated from equation 2 . The vector $\vec{\omega}_{\text {ball }}$ is in the opposite direction of $\vec{\omega}_{\text {hand }}$. In this equation, $\overrightarrow{C A}$ is the position vector of the geometric centre $\mathrm{A}$ of the ball with respect to point $\mathrm{C}$. This vector, given by equation 3 , is considered to be perpendicular to $\vec{\omega}_{\text {hand }}=[\omega$ hand,$x \omega$ hand,y 0$]$ and depends on the angle $\varphi$ (Figure 2). Its norm $r$ is equal to the radius of the ball $(0.125 \mathrm{~m})$. The release angle with respect to the horizontal $\theta_{\text {release }}$ was computed using equation 4 .

$$
\begin{gathered}
\vec{v}_{\text {release }}=\vec{v}_{\text {fingers }}+\vec{\omega}_{\text {ball }} \overrightarrow{C A} \\
\overrightarrow{C A}=\left[\begin{array}{lll}
r \cos (\phi) \omega_{\text {hand, } y} & \frac{-r \cos (\phi) \omega_{\text {hand } x}}{\sqrt{\omega_{\text {hand }, x}^{2}+\omega_{\text {hand }, x}^{2}}} & r \sin (\phi) \\
\sqrt{\omega_{\text {hand }, x}^{2}+\omega_{\text {hand }, x}^{2}}
\end{array}\right] \\
\theta_{\text {release }}=\tan ^{-1} \frac{v_{z}}{\sqrt{v_{x}^{2}+v_{y}^{2}}}
\end{gathered}
$$


where $v x, v_{y}$ and $v_{z}$ are respectively the $x, y$, and $z$ components of $\vec{v}_{\text {release }}$.

One combination of release variables ( $\left.v_{\text {release and }} \theta_{\text {release}}\right)$ was computed for each possible combination of $(\varphi, \omega$ ball $)$ for $\varphi$ varying from $20\left(^{\circ} \cdot \mathrm{s}^{-}\right.$ 1) to $80\left({ }^{\circ} \cdot \mathrm{s}^{-1}\right)$ and for $\omega$ ball varying between $0\left({ }^{\circ} \cdot \mathrm{s}^{-1}\right)$ and $80\left({ }^{\circ} \cdot \mathrm{s}^{-1}\right)$, where $v_{\text {release }}$ and $\omega_{\text {ball }}$ are the norms of $\vec{v}_{\text {release }}$ and $\vec{\omega}_{\text {ball }}$ respectively. In 3PS, typical values for $v_{\text {release }}$ and $\theta_{\text {release }}$ were found to range from $5 \mathrm{~m} / \mathrm{s}$ to $6 \mathrm{~m} / \mathrm{s}$ and $52^{\circ}$ to $57^{\circ}$, respectively (Miller and Bartlett, 1996). Only the values of $\vec{v}_{\text {release }}$ and $\theta_{\text {release }}$ that corresponded to typical values for 3PS were registered, along with the corresponding ranges for $\varphi$ and $\omega$ ball.

\section{Statistical analyses}

All data were expressed as means \pm standard deviations (SD). A paired student t-test (pre vs. post fatigue) was used to analyse the effects of fatigue on the kinematics of the CoM (vertical displacement), joint angles (at time of the lowest CoM position and time of ball release) and ball release variables. A paired t-test was also used to compare $H_{\max }$ obtained during ExEx (first session) with $\mathrm{HR}_{\max }$ reached during the fatigue protocol, and to compare the time of the first and last $20 \mathrm{~m}$ sprints. For all statistical analyses, a $p$-value of 0.05 was considered to indicate significance.

\section{Results}

\section{Exercise protocol}

During the first session, mean TExEx was $142.8 \pm 62.6 \mathrm{~s}$ and mean HRex was $182.1 \pm 9.4 \mathrm{bpm}$.

During the second session, the mean time to reach $70 \%$ of the time to exhaustion ( $\mathrm{T}_{\text {ExEx70\%) }}$ was $99.7 \pm 43.9$ s. Performance time of the last $20 \mathrm{~m}$ sprint during the ExEx until $70 \%$ of TExEx, was significantly longer than the first sprint $(3.85 \pm 0.24$ $\mathrm{s}$ vs $4.50 \pm 0.77 \mathrm{~s} ; p \leq 0.05)$. Mean $\mathrm{HR}$ value at $\mathrm{T}_{\mathrm{ExEx} 70 \%}$ was $179.1 \pm 8.4 \mathrm{bpm}$, which was $98 \%$ of HRex and $88 \%$ of the theoretical maximal heart rate. The Borg scale score was $15.0 \pm 1.7$. The maximal HR value reached for $\mathrm{T}_{\mathrm{ExEx} 70 \%}$ was significantly lower than $\operatorname{HR}_{\text {Ex }}(p \leq 0.05)$.

\section{3-points shooting drill}

Wrist, elbow, shoulder, hip, knee, and ankle angles for abduction/adduction, internal/external and flexion/extension movements are presented in Table 1 (lowest position of the CoM) and Table 2 (time of ball release). There were no significant differences between any of the joint angles recorded pre and post exercise until $70 \%$ of T $\operatorname{ExEx}(p$ $\geq 0.09$ ). Ball release variables and vertical displacement of the CoM also did not differ between the two conditions (Table 3; $p \geq 0.14$ ).

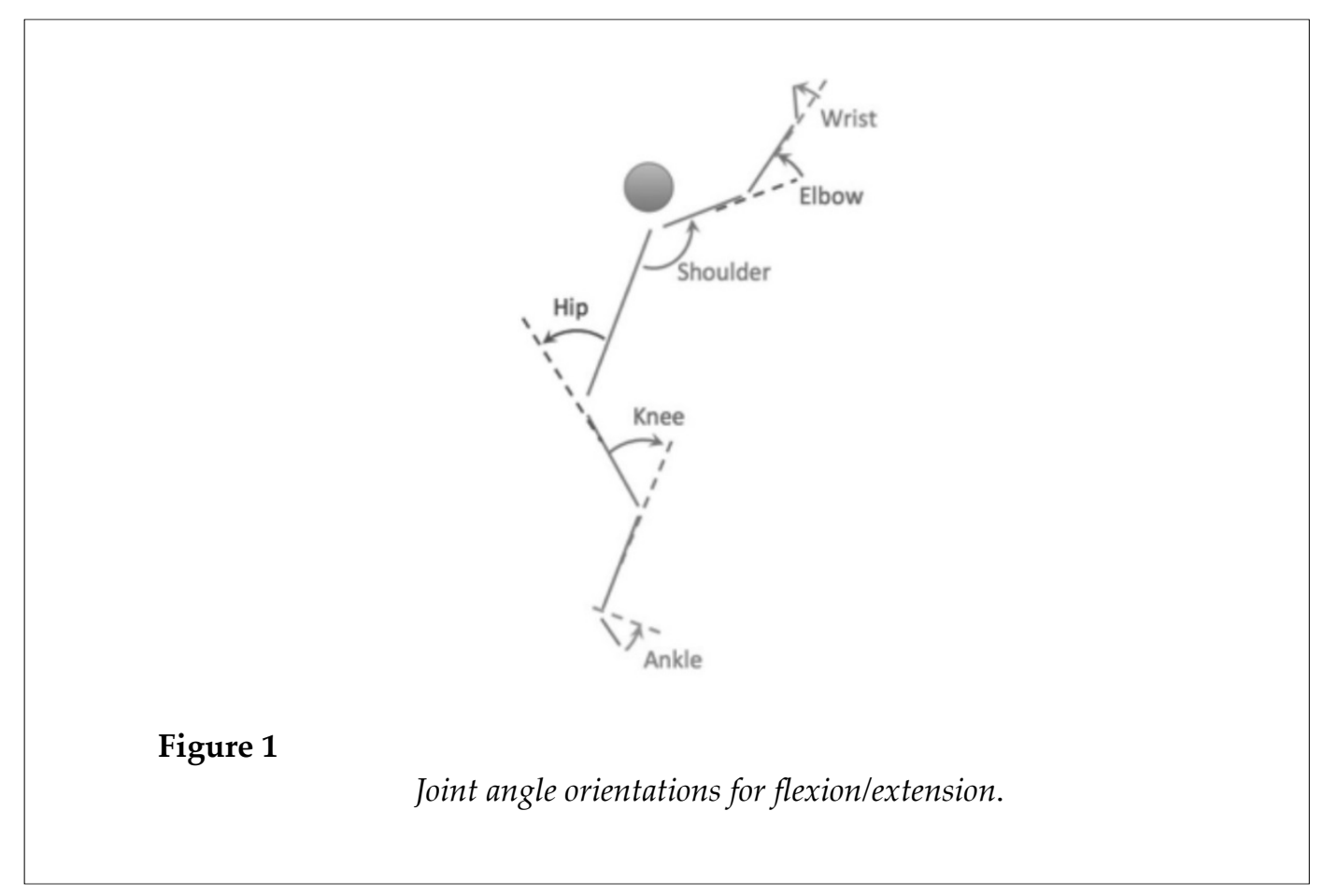




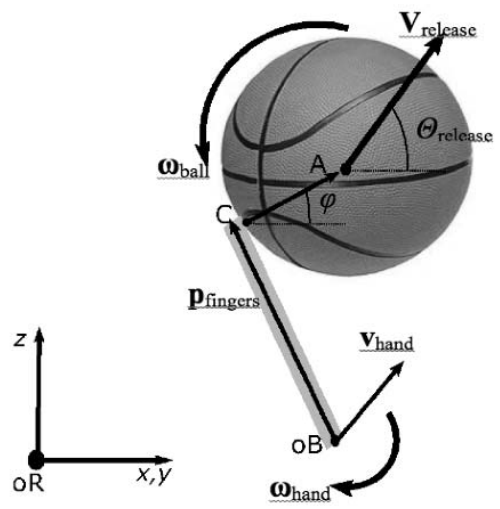

Figure 2

The hand and fingers were modeled as a rigid segment $B C$ and kinematics were calculated at trelease. The ball was released from point $C$ with velocity vrelease, angle $\theta_{\text {release, }}$ angular velocity wball and angle $\phi$.

\begin{tabular}{|c|c|c|c|c|}
\hline \multicolumn{3}{|c|}{$\begin{array}{l}\text { Table } 1 \\
\text { Shoulder, elbow, wrist, hip, knee, and ankle angles at ball reception } \\
\text { (centre of mass at its lowest point). } \\
\text { sitive values represent abduction, internal rotation or flexion movements } \\
\text { and negative values represent adduction, extension or external rotation. }\end{array}$} & \multicolumn{2}{|c|}{$\begin{array}{l}\text { Table } 1 \\
\text { ball reception } \\
\text { flexion movements } \\
\text { external rotation. }\end{array}$} \\
\hline & & Non-Fatigue & Fatigue & $p$ \\
\hline \multirow[t]{3}{*}{ Shoulder $\left(^{\circ}\right)$} & $A b d+/ A d d-$ & $22.0 \pm 10.1$ & $22.2 \pm 10.0$ & 0.81 \\
\hline & $I R+/ E R-$ & $8.9 \pm 14.3$ & $7.6 \pm 12.4$ & 0.49 \\
\hline & Flex+/Ext- & $48.7 \pm 16.6$ & $44.2 \pm 12.6$ & 0.26 \\
\hline \multirow[t]{2}{*}{ Elbow $\left({ }^{\circ}\right)$} & $I R+/ E R-$ & $112.3 \pm 23.3$ & $111.2 \pm 23.4$ & 0.49 \\
\hline & Flex+/Ext- & $101.2 \pm 13.9$ & $100.8 \pm 14.6$ & 0.81 \\
\hline \multirow[t]{2}{*}{ Wrist $\left({ }^{\circ}\right)$} & $A b d+/ A d d-$ & $19.7 \pm 19.4$ & $17.3 \pm 17.8$ & 0,46 \\
\hline & Flex+/Ext- & $0.2 \pm 10.3$ & $5.0 \pm 17.5$ & 0.45 \\
\hline \multirow[t]{3}{*}{$\operatorname{Hip}\left({ }^{\circ}\right)$} & $A b d+/ A d d-$ & $3.8 \pm 4.9$ & $4.2 \pm 7.6$ & 0.72 \\
\hline & $I R+/ E R-$ & $9.0 \pm 7.1$ & $9.7 \pm 7.0$ & 0.72 \\
\hline & Flex+/Ext- & $48.3 \pm 10.6$ & $46.3 \pm 10.9$ & 0.28 \\
\hline \multirow[t]{2}{*}{ Knee $\left(^{\circ}\right)$} & $I R+/ E R-$ & $3.6 \pm 8.4$ & $2.9 \pm 5.5$ & 0.57 \\
\hline & Flex+/Ext- & $77.6 \pm 8.7$ & $77.5 \pm 12.8$ & 0.96 \\
\hline \multirow[t]{2}{*}{ Ankle $\left(^{\circ}\right)$} & $A b d+/ A d d-$ & $-4.3 \pm 8.5$ & $-1.7 \pm 7.1$ & 0.30 \\
\hline & Flex+/Ext- & $27.1 \pm 7.4$ & $27.5 \pm 7.0$ & 0.65 \\
\hline
\end{tabular}


Table 2

Shoulder, elbow, wrist, hip, knee, and ankle angles at ball release.

Positive values represent abduction, internal rotation or flexion movements and negative values represent adduction, extension or external rotation.

\begin{tabular}{clccc}
\hline & & Non-Fatigue & Fatigue & $p$ \\
\hline Shoulder $\left(^{\circ}\right)$ & Abd+/Add- & $43.6 \pm 8.1$ & $44.3 \pm 9.9$ & 0.39 \\
& IR+/ER- & $45.7 \pm 17.8$ & $48.4 \pm 21.4$ & 0.09 \\
& Flex+/Ext- & $114.7 \pm 16.4$ & $118.5 \pm 13.3$ & 0.33 \\
Elbow $\left(^{\circ}\right)$ & IR+/ER- & $109.7 \pm 35.4$ & $108.8 \pm 19.9$ & 0.57 \\
& Flex+/Ext- & $28.1 \pm 30.7$ & $21.9 \pm 13.6$ & 0.14 \\
Wrist $\left(^{\circ}\right)$ & Abd+/Add- & $13.0 \pm 31.0$ & $10.6 \pm 18.1$ & 0.33 \\
& Flex+/Ext- & $-41.2 \pm 31.9$ & $-41.2 \pm 21.5$ & 0.57 \\
Hip $\left(^{\circ}\right)$ & Abd+/Add- & $4.1 \pm 4.1$ & $3.3 \pm 6.0$ & 0.65 \\
& IR+/ER- & $-5.7 \pm 11.1$ & $-7.0 \pm 10.5$ & 0.72 \\
& Flex+/Ext- & $-2.4 \pm 9.9$ & $-1.8 \pm 10.5$ & 0.39 \\
Knee $\left({ }^{\circ}\right)$ & IR+/ER- & $1.5 \pm 8.6$ & $0.7 \pm 6.3$ & 0.55 \\
& Flex+/Ext- & $19.6 \pm 8.2$ & $18.5 \pm 10.7$ & 0.62 \\
Ankle $\left(^{\circ}\right)$ & Abd+/Add- & $-16.1 \pm 8.7$ & $-14.6 \pm 5.8$ & 0.59 \\
& Flex+/Ext- & $-29.1 \pm 8.5$ & $-28.5 \pm 8.4$ & 0.74 \\
\hline
\end{tabular}

Table 3

Maximal vertical displacement of the centre of mass (CM) during the shot, and ball release variables.

\begin{tabular}{lllc}
\hline & Non Fatigue & Fatigue & $P$ \\
\hline Vertical displacement of the CM $(\mathrm{m})$ & $0.39 \pm 0.06$ & $0.39 \pm 0.04$ & 0.96 \\
Release velocity $\left(\mathrm{m} \cdot \mathrm{s}^{-1}\right)$ & $7.77 \pm 0.20$ & $7.80 \pm 0.13$ & 0.8 \\
Release angle $\left(^{\circ}\right)$ & $51.1 \pm 0.4$ & $51.0 \pm 0.2$ & 0.14 \\
Release height $(\mathrm{m})$ & $2.35 \pm 0.24$ & $2.31 \pm 0.21$ & 0.51 \\
\hline
\end{tabular}

\section{List of abbreviations}

ExEx: The first volitional exhausting exercise; TExEx: Time to exhaustion of the first volitional exhausting

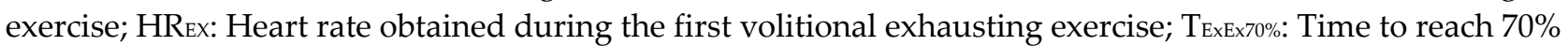
of the exhaustion of the first volitional exhausting exercise. This variable was the duration of the second exercise; CoM: the centre of mass; trelease: Release time; $\vec{v}_{\text {fingers }}:$ Velocity of the fingers; $\vec{v}_{\text {wrist }}:$ Wrist velocity; $\vec{\omega}_{\text {hand }}$ : hand angular velocity; $\vec{p}_{\text {fingers }}$ : length and orientation of the fingers; $\vec{\omega}_{\text {ball }}$ : Ball's angular velocity;

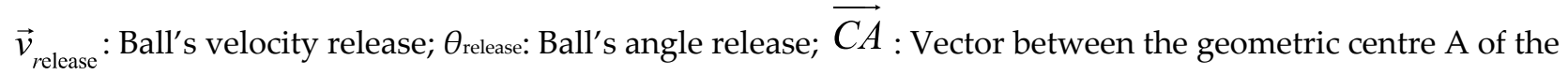
ball and the contact point with the ball; $\varphi$ : Angle between $\overrightarrow{C A}$ and the horizontal; 
This study investigated the effects of fatigue on the kinematics of 3PS in basketball. The 3D angles of the hip, knee and ankle when the centre of mass was at its lowest point and the 3D angles of the hip, shoulder, elbow and wrist when the ball was released were compared. The release variables of the ball were estimated by modeling the release gesture. There was no effect of physical fatigue on the joint angles of any of the body segments involved in 3PS, or on the ball release variables.

One of the main challenges of the present study was to produce similar physical fatigue to that which occurs during a real basketball game. Although it is difficult to precisely predict the level of fatigue which occurs, it is well known that a basketball game elicits from around $80 \%$ to $90 \%$ of the maximal heart rate (Ben Abdelkrim et al., 2007; Erculj and Supej, 2009; McInnes et al., 1995). Accordingly, we designed a fatigue protocol which would produce $80-90 \%$ of the maximal HR. HR at the end of the fatiguing exercise was $179 \pm 8.4 \mathrm{bpm}$, corresponding to $88 \%$ of the theoretical HRmax. Performance time increased significantly from the first to the last $20 \mathrm{~m}$ sprint during the second session of the fatiguing exercise protocol during which 3PS was recorded. This demonstrates that the players were unable to maintain their sprint velocity and that they reached a fatigued state. Moreover, we deliberately used an exercise protocol that included several important aspects of a basketball game (i.e. sprinting, jumping, accelerating and decelerating) to mimic the repeated sprints that occur during a game (Ben Abdelkrim et al., 2007; McInnes et al., 1995). Uygur et al. (2010) recorded similar values in collegiate level male basketball players (mean $\mathrm{HR}=175.1$ bpm corresponding to $85-90 \%$ of HRmax) using a similar exercise protocol. Another study used the Basketball Exercise Simulation Test (BEST) fatiguing protocol and found that it induced 90 to 92\% of the theoretical HRmax (calculated from HRmax $=220-$ age) (Scanlan et al., 2012). In addition, in the present study, the average time to exhaustion (142.8 $\pm 62.6 \mathrm{~s})$ was in accordance with a previous study (Uygur et al., 2010), confirming that our participants truly reached exhaustion and could no longer run and jump when they stopped the test. Reaching an appropriate level of fatigue was

a prerequisite in order to study accurately the effects of fatigue typically encountered during a basketball game.

The biomechanical analysis revealed that the exercise-induced fatigue had no effect on any of the kinematic variables measured or estimated during 3PS. To the best of our knowledge, the present study is the first to report the effects of fatigue on kinematic motion variables in basketball by using nanotechnology inertial motion units directly attached to body segments. This modern technology provides a new insight into factors which influence the accuracy of 3PS by recording data directly 'on the field'. Indeed, because of its small size and light weight (less than $2 \mathrm{~kg}$ ), the inertial suit did not interfere with the players' movements and could be used directly in their usual training environment. A previous study found a significant decrease in jump height and the elbow joint angle, and an increase in the shoulder joint angle with fatigue using 3D video analysis (Erculj and Supej, 2009). In the present study, we only observed a trend towards an increase in the shoulder joint angle with fatigue. There was no change in jump height or elbow angle. These differences likely relate to two factors. The first is the time at which the different biomechanical variables were recorded. Previous studies analysed joint variables $\sim 60 \mathrm{~ms}$ before ball release (Erculj and Supej, 2009) and at 3 different time points: ball release, and $83.3 \mathrm{~ms}$ (5 frames) before and after ball release (Uygur et al., 2010). The use of only wearable inertial sensors in the present study did not allow precise determination of the release point. We thus assumed that the moment of release occurred when the orientation of the hand was close to vertical. Thus the biomechanical analysis was not carried out at the same time points in the different studies. The second factor relates to the level of fatigue experienced by the players. Our fatigue protocol was similar to that of Uygur et al. (2010), inducing around $88 \%$ of the players' theoretical HRmax. In contrast, the fatigue protocol used in a previous study (Erculj and Supej, 2009) (6 sets of $8 \times 10 \mathrm{~s}$ sprints, direction changes and shots) induced $97 \%$ of the theoretical HRmax. As inferred through HR values, the protocol used in the latter study was probably much more fatiguing than in the present study and might have led to significant alterations of the shooting kinematics. Ball release variables have been identified as determinants of 3PS performance in 
basketball (Okazaki et al., 2015). Release velocity, angle and height have been widely studied. Ball release variables depend on the preparation phases for the shot, and all the kinematics involved. A jump shot consists of 5 phases (i.e. preparation, ball elevation, stability, release, inertia) during which all body segments are mobilized to produce optimal ball release (Okazaki et al., 2015). Taken together, data from the literature suggest that height release, which allows a lower movement velocity and release angle, is the main factor related to a successful shot (Hamilton and Reinschmidt, 1997; Malone et al., 2002; Miller and Bartlett, 1993). Ankle, knee, hip, shoulder, elbow and wrist joint angles during the ball release phase were recently analysed by using a $100 \mathrm{~Hz}$ video camera (Okazaki and Rodacki, 2012). Interestingly, the hip and knee angles recorded in the present study are similar to those reported by Okazaki and Rodacki (2012). However, the elbow $\left(165^{\circ}\right)$ and wrist $\left(221^{\circ}\right)$ angles at the time of ball release were greater $\left(+30^{\circ}\right)$ in our study than in theirs. This difference is likely due to the different methods of determination of ball release, i.e. the use of inertial technology in the present study (vs. a video camera) did not allow the visualisation of ball release. However, despite this potential limitation, the ball release variables we calculated are in accordance with the literature. For example, for a $6.75 \mathrm{~m} \mathrm{3}$-point shot, ball release velocity in the literature is generally between 6.67 and $6.89 \mathrm{~m} \cdot \mathrm{s}-1$ (around $7.8 \mathrm{~m} \cdot \mathrm{s}-1$ in the present study), release angle is between 49 and $60^{\circ}\left(\sim 51^{\circ}\right.$ in the present study), and ball release height is between 2.05 and $2.77 \mathrm{~m} \mathrm{(} \sim 2.30 \mathrm{~m}$ in the present study) (Mullineaux and Uhl, 2010; Okazaki and Rodacki, 2012). Such small differences between the present results and the literature support the absence of significant effect of fatigue on kinematic variables.

Considering the complexity of 3PS, analysis of the effects of physical fatigue on kinematics is challenging. Scoring in basketball requires both motor abilities (strength, speed, agility) and basketball specific skills (Okazaki et al., 2015). Muscle strength and power also determine ball release variables, while coordination and good balance are key abilities of elite basketball players (Hudson, 1985; Uygur et al., 2010). One hypothesis tested in the present study was that physical fatigue induced by a specific basketball-based repeated sprint protocol could have a deleterious effect on muscle variables, neural factors and stiffness regulation, altering the kinematics of the shot. However, our results refute this hypothesis, showing that players well tolerated the effects of fatigue during 3PS. The high level and experience of players included may explain why the kinematic variables were so stable, despite exercise-induced fatigue. It would be reasonable to suggest that the players had automated the optimal kinematics required for 3PS so that they were able to maintain good stability, good balance, and high ball release with minimal effort. Several studies have reported greater consistency of the kinematic patterns of free throw shooters who had more playing experience compared with less experienced shooters (Button et al., 2003; Okazaki and Rodacki, 2012). In contrast, novice players generally adopt a "freezing" strategy in order to simplify the cognitive demand (i.e. controlling the ball while focusing on the target) relating to the action (Okazaki and Rodacki, 2012). However this strategy reduces the range of motion of all joints and the possibility of using alternative strategies such as counter-movement around the elbow (Okazaki and Rodacki, 2012). In accordance with the literature, results from the present study suggest that the shooting performance of elite basketball players is highly consistent, as demonstrated by the stability of their kinematic profiles.

The results of this study must be considered in the context of the limitations in the study design. The 3PS depends more on skills than physical capacity, likely explaining why there was no change in kinematics between the non-fatigued and fatigued states (Hudson, 1985). It is possible that the results would have been different in a more physically interactive situation, such as with an opponent. During a match, the complex interaction between fatigue, tactical decisions, score-line, time remaining and defensive pressure, may also influence the kinematics and accuracy of 3PS, particularly in young players (Malarranha et al., 2013). Fatigue is not an isolated factor that modifies the execution of a shot. However, the purpose of the present study was to attempt to understand the effect of fatigue on the kinematics of the shot, thus to answer this question it was necessary to consider only fatigue without other confounding factors such as pressure from 
opponents and time, or interactive situations.

The present study demonstrated that repeated sprints and jumps did not affect the kinematics of 3PS in young elite players. From a practical point of view this result suggests that shooting exercises can be carried out either at the beginning or the end of a training session. The fatigue induced by a training session will not modify kinematics and therefore, will probably not be associated with a high risk of injury. It appears that young players learn to cope with physical fatigue while performing coordinated movements. Accordingly, shooting training should be carried out in a fatigued state so that

less-experienced players can learn to cope with fatigue and still produce coordinated movements.

The ability to carry out successful 3PS is a determinant of performance in modern basketball.
The purpose of this study was to analyze the effects of physical fatigue on the kinematic variables of 3PS in elite basketball players. Particular attention was paid to reproducing playing conditions similar to those of a real basketball game; i. the exercise protocol was designed to mimic the physical demands of a basketball game; ii. motion kinematics were recorded using nanotechnology inertial measurement units, that were directly attached to all body segments, and did not interfere with the players' movements. The results showed that there was no effect of fatigue on any of the main joints involved in the movement (i.e. wrist, shoulder, elbow, hip, knee, and ankle), either when the centre of mass was at its lowest position, or at ball release. In addition, ball release variables were not modified by the fatigue protocol. Our results suggest that physical fatigue induced during a match does not alter kinematic variables of 3PS in elite basketball players.

\section{Acknowledgements}

We greatly thank Sinisa Karganovic and Richard Billand, head coach of the basketball section at the French National Institute of Sports. We also express gratitude to the players who took part in the study.

\section{References}

Ben Abdelkrim N, Castagna C, Jabri I, Battikh T, El Fazaa S, El Ati J. Activity profile and physiological requirements of junior elite basketball players in relation to aerobic-anaerobic fitness. J Strength Cond Res, 2010; 24(9): 2330-42

Ben Abdelkrim N, El Fazaa S, El Ati J. Time-motion analysis and physiological data of elite under-19-year-old basketball players during competition. Br J Sports Med, 2007; 41(2): 69-75

Bar-Eli M, Avugos S, Raab M. Twenty years of "hot hand" research: Review and critique. Psychol Sport Exerc, 2006;7(6): 525-53

Borg GA. Psychophysical bases of perceived exertion. Med Sci Sports Exerc, 1982; 14(5): 377-81

Button C, MacLeod M, Sanders R, Coleman S. Examining movement variability in the basketball free-throw action at different skill levels. Res. Q. Exerc. Sport, 2003; 74(3): 257-69

Chappell JD. Effect of Fatigue on Knee Kinetics and Kinematics in Stop-Jump Tasks. Am. J. Sports Med, 2005; 33(7): 1022-9

Delextrat A, Baliqi F, Clarke N. Repeated sprint ability and stride kinematics are altered following an official match in national-level basketball players. J Sports Med Phys Fitness, 2013; 53(2): 112-8

Erculj F, Supej M. Impact of fatigue on the position of the release arm and soulder girdle over a longer shooting distance for elite basketball player. J Strength Cond Res, 2009; 23(3): 1029-36

Girard O, Mendez-Villanueva A, Bishop D. Repeated-sprint ability - part I: factors contributing to fatigue. Sports Med, 2011; 41(8): 673-94

Guo D, Fei Deng F, Zhang Z. 3-point shot performance analysis. Athens, pre-olympic Congr. Sport Sci Through Ages: Challenges in New Millenn, 2; 2004

Hamilton GR, Reinschmidt C. Optimal trajectory for the basketball free throw. J Sports Sci, 1997; 15(5): 491504

Hudson JL. Prediction of basketball skill using biomechanical variables. Res Q Exerc Sport, 1985; 56: 115-21 
Lyons M, Al-Nakeeb Y, Nevill A. The impact of moderate and high intensity total body fatigue on passing accuracy in expert and novice basketball players. J Sports Sci Med, 2006; 5(2): 215-27

Malarranha J, Figueira B, Leite N, Sampaio J. Dynamic Modeling of Performance in Basketball. Int J Perform Anal Sport, 2013; 13(2): 377-87

Malone LA, Gervais PL, Steadward RD. Shooting mechanics related to player classification and free throw success in wheelchair basketball. J Rehabil Res Dev, 2002; 39(6): 701-9

Matthew D, Delextrat A. Heart rate, blood lactate concentration, and time-motion analysis of female basketball players during competition. J Sports Sci, 2009; 27(8): 813-21

McInnes SE, Carlson JS, Jones CJ, McKenna MJ. The physiological load imposed on basketball players during competition. J Sports Sci, 1995; 13(5): 387-97

Meriam JL, Kraige LG. Mechanics-Dynamics 7th Edition Binder Ready Version edition; 2012

Mikolajec K, Waskiewicz Z, Maszczyk A, Bacik B, Kurek P, Zając A. Effects of stretching and strength exercises on speed and power abilities in male basketball players. Isokinet Exer Sci, 2012; 20(1): 61-69

Miller S, Bartlett R. The relationship between basketball shooting kinematics, distance and playing position. J Sports Sci, 1996; 14(3): 243-53

Miller S, Bartlett RM. The effects of increased shooting distance in the basketball jump shot. J Sports Sci, 1993; 11(4): 285-93

Montgomery PG, Pyne DB, Minahan CL. The physical and physiological demands of basketball training and competition. Int J Sports Physiol Perform, 2010 Mar; 5(1): 75-86

Mullineaux DR, Uhl TL. Coordination-variability and kinematics of misses versus swishes of basketball free throws. J Sports Sci, 2010; 28(9): 1017-24

Okazaki VHA, Rodacki ALF. Increased distance of shooting on basketball jump shot. J Sports Sci Med, 2012; 11(2): 231-7

Okazaki VH, Rodacki ALF, Satern MN. A review on the basketball jump shot. Sport Biomech, 2015; 14(2): 190205

Padulo J, Laffaye G, Haddad M, Chaouachi A, Attene G, Migliaccio GM, Chamari K, Pizzolato F. Repeated sprint ability in young basketball players: one vs. two changes of direction (Part 1). J Sports Sci, 2015; 33(14): 1480-92

Rodacki AL, Fowler NE, Bennett SJ. Vertical jump coordination: fatigue effects. Med Sci Sport, Exerc, 2002; 34(1): 105-16

Rojas FJ, Cepero M, Oña A, Gutierrez M. Kinematic adjustments in the basketball jump shot against an opponent. Ergonomics, 2000; 43(10): 1651-60

Scanlan AT, Dascombe BJ, Reaburn PR. The Construct and Longitudinal Validity of the Basketball Exercise Simulation Test. J Strength Cond Res, 2012; 26(2): 523-30

Uygur M, Goktepe A, Ak E, Karabörk H, Korkusuz F. Effect of Fatigue on the Kinematics of Free Throw Shooting. J Hum Kinet, 2010; 24: 51-6

\section{Corresponding author:}

\section{Jean Slawinski, PhD}

Centre de recherche sur le sport et le mouvement - EA 2931.

Université de Paris Ouest Nanterre La Défense

200 avenue de la république 92000 Nanterre, France.

Phone:+331 40975630

E-mail: jslawinski@parisnanterre.fr 